\title{
Velocity Characteristics of Vertical Buoyant Jet from a Square Orifice in Static Ambient
}

\author{
Wang Shaobo ${ }^{1, a,{ }^{*}}$, Sun Xiaoli, ${ }^{2, b}$ and Xie Yufeng ${ }^{1, c}$ \\ ${ }^{1}$ Pearl River Hydraulic Research Institute, Guangzhou 510611, China \\ ${ }^{2}$ Pearl River Water Resources Commission of the Ministry of Water Resources, Guangzhou 510611, \\ China \\ a83202208@qq.com, b38613211@qq.com, '1075431956@qq.com \\ *corresponding author
}

Key words: buoyant jet, square orifice, numerical simulation, static ambient

\begin{abstract}
A three-dimensional eddy model considering buoyancy was established to simulate vertical buoyant flow from a square orifice in static ambient. The hybrid finite analytic method with stagged grid was used to solve this model. Results showed that axial velocities from a square orifice coincided well with those from a circular one after a little distance from the jet mouth. The shapes of vertical velocity distribution on different height cross-sections tallied well with those from a circular orifice. The existence of self-similar area of longitudinal velocity was confirmed also.
\end{abstract}

\section{Introduction}

There are lots of fruits about characteristics of vertical buoyant jets from a circular orifice existed by previous scholars. Systemic review and summarization on round vertical turbulent buoyant jets have been made by List $(1982)^{[1]}$ and Chen and Rodi $(1980)^{[2]}$. Extensive reviews have been reported by Jirka, Abraham and Harleman $(1975)^{[3]}$ as well as by Fischer et al. (1979) ${ }^{[4]}$. Unified formulas of axial concentration and velocity for jets with or not buoyancy have been proposed by Noutsopoulos and Yannopoulos $(1987)^{[5]}$, in which integral forms of the momentum and tracer equations were integrated on the basis of an assumption concerning a function of the spreading coefficients. The dilution characteristics of obstructed buoyant vertical jets in static ambient were also studied by Huai et al (2006) ${ }^{[6]}$ and Huai and Fang (2006) ${ }^{[7]}$. However, papers about vertical turbulent buoyant jets from a square orifice are rather less published and whether characteristics of them may be similar to those of jets from a round one needs more researching effort. Numerical simulations have been made on vertical turbulent buoyant jets of thermal water from a square orifice in static ambient. Reliability and efficiency of the mathematical model used have been verified, and results from numerical simulations were compared with those of jets from a round orifice with its diameter equals to side length of the square orifice.

\section{Mathematical Model}

Dimensionless turbulent RNG $k-\varepsilon$ mathematical model is as following based on characteristic quantities of exit velocity $v_{j}$, side length of the square orifice $d$ and ambient water density $\rho_{a}$ :

$$
\begin{gathered}
\frac{\partial u}{\partial x}+\frac{\partial v}{\partial y}+\frac{\partial w}{\partial z}=0 \\
\operatorname{Re}_{e t}\left(u-2 \frac{\partial v_{t}}{\partial x}\right) \frac{\partial u}{\partial x}+\operatorname{Re}_{e t}\left(v-\frac{\partial v_{t}}{\partial y}\right) \frac{\partial u}{\partial y}+\operatorname{Re}_{e t}\left(w-\frac{\partial v_{t}}{\partial z}\right) \frac{\partial u}{\partial z}= \\
\left(\frac{\partial^{2} u}{\partial x^{2}}+\frac{\partial^{2} u}{\partial y^{2}}+\frac{\partial^{2} u}{\partial z^{2}}\right)+\operatorname{Re}_{e t}\left(-\frac{\partial p}{\partial x}-\frac{2}{3} \frac{\partial k}{\partial x}+\frac{\partial v_{t}}{\partial y} \frac{\partial v}{\partial x}+\frac{\partial v_{t}}{\partial z} \frac{\partial w}{\partial x}\right)
\end{gathered}
$$




$$
\begin{gathered}
\operatorname{Re}_{e t}\left(u-\frac{\partial v_{t}}{\partial x}\right) \frac{\partial v}{\partial x}+\operatorname{Re}_{e t}\left(v-2 \frac{\partial v_{t}}{\partial y}\right) \frac{\partial v}{\partial y}+\operatorname{Re}_{e t}\left(w-\frac{\partial v_{t}}{\partial z}\right) \frac{\partial v}{\partial z}= \\
\left(\frac{\partial^{2} v}{\partial x^{2}}+\frac{\partial^{2} v}{\partial y^{2}}+\frac{\partial^{2} v}{\partial z^{2}}\right)+\operatorname{Re}_{e t}\left(-\frac{\partial p}{\partial y}-\frac{2}{3} \frac{\partial k}{\partial y}+\frac{\partial v_{t}}{\partial x} \frac{\partial u}{\partial y}+\frac{\partial v_{t}}{\partial z} \frac{\partial w}{\partial y}\right)+\beta g \Delta T \frac{d}{v_{j}^{2}} \\
\operatorname{Re}_{e t}\left(u-\frac{\partial v_{t}}{\partial x}\right) \frac{\partial w}{\partial x}+\operatorname{Re}_{e t}\left(v-\frac{\partial v_{t}}{\partial y}\right) \frac{\partial w}{\partial y}+\operatorname{Re}_{e t}\left(w-2 \frac{\partial v_{t}}{\partial z}\right) \frac{\partial w}{\partial z}= \\
\left(\frac{\partial^{2} w}{\partial x^{2}}+\frac{\partial^{2} w}{\partial y^{2}}+\frac{\partial^{2} w}{\partial z^{2}}\right)+\operatorname{Re}_{e t}\left(-\frac{\partial p}{\partial z}-\frac{2}{3} \frac{\partial k}{\partial z}+\frac{\partial v_{t}}{\partial x} \frac{\partial u}{\partial z}+\frac{\partial v_{t}}{\partial y} \frac{\partial v}{\partial z}\right) \\
\operatorname{Re}_{e k}\left(u-\frac{1}{\sigma_{k}} \frac{\partial v_{t}}{\partial x}\right) \frac{\partial k}{\partial x}+\operatorname{Re}_{e k}\left(v-\frac{1}{\sigma_{k}} \frac{\partial v_{t}}{\partial y}\right) \frac{\partial k}{\partial y}+\operatorname{Re}_{e t}\left(w-\frac{1}{\sigma_{k}} \frac{\partial v_{t}}{\partial z}\right) \frac{\partial k}{\partial z}= \\
\left(\frac{\partial^{2} k}{\partial x^{2}}+\frac{\partial^{2} k}{\partial y^{2}}+\frac{\partial^{2} k}{\partial z^{2}}\right)+\operatorname{Re}_{e k}\left(P_{k}-\varepsilon+\alpha g \frac{v_{t}}{P_{r t}} \frac{\partial T}{\partial z} \frac{d}{v_{j}^{2}}\right) \\
\operatorname{Re}_{e \varepsilon}\left(u-\frac{1}{\sigma_{k}} \frac{\partial v_{t}}{\partial x}\right) \frac{\partial \varepsilon}{\partial x}+\operatorname{Re}_{e \varepsilon}\left(v-\frac{1}{\sigma_{k}} \frac{\partial v_{t}}{\partial y}\right) \frac{\partial \varepsilon}{\partial y}+\operatorname{Re}_{e \varepsilon}\left(w-\frac{1}{\sigma_{k}} \frac{\partial v_{t}}{\partial z}\right) \frac{\partial \varepsilon}{\partial z}= \\
\left(\frac{\partial^{2} \varepsilon}{\partial x^{2}}+\frac{\partial^{2} \varepsilon}{\partial y^{2}}+\frac{\partial^{2} \varepsilon}{\partial z^{2}}\right)+\operatorname{Re}_{e \varepsilon}\left(C_{\varepsilon 1} \frac{\varepsilon}{k}\left(P_{k}+\alpha g \frac{v_{t}}{P_{r t}} \frac{\partial T}{\partial y} \frac{d}{v_{j}^{2}}\right)-C_{\varepsilon 2} \frac{\varepsilon^{2}}{k}\right) \\
\operatorname{Re}_{e T}\left(u-\frac{1}{\mathrm{P}_{r t}} \frac{\partial v_{t}}{\partial x}\right) \frac{\partial T}{\partial x}+\operatorname{Re}_{e T}\left(v-\frac{1}{\mathrm{P}_{r t}} \frac{\partial v_{t}}{\partial y}\right) \frac{\partial T}{\partial y}+\operatorname{Re}_{e t}\left(w-\frac{1}{\mathrm{P}_{r t}} \frac{\partial v_{t}}{\partial z}\right) \frac{\partial T}{\partial z}= \\
\left(\frac{\partial^{2} T}{\partial x^{2}}+\frac{\partial^{2} T}{\partial y^{2}}+\frac{\partial^{2} T}{\partial z^{2}}\right)
\end{gathered}
$$

where $x$ and $z$ denote horizontal directional coordinate and $y$ vertical directional coordinate. $u, v$ and $w$ are velocities in $x, y$ and $z$ direction. $p, k, \varepsilon, T$ denote pressure, turbulent kinetic energy, energy dissipation rate and temperature respectively. $P_{r}$ and $P_{r t}$ are Prandtl numbers with value of 0.7 used here. $v$ and $v_{t}$ are kinetic viscosity coefficients. $R_{e t}, R_{e k}, R_{e \varepsilon}$ and $R_{e T}$ are dimensionless coefficients in deducing above equations and expressed as following:

$$
R_{e t}=\frac{\operatorname{Re}}{1+\operatorname{Re} v_{t}}, \quad R_{e k}=\frac{\operatorname{Re}}{1+\operatorname{Re} v_{t} / \sigma_{k}}, \quad R_{e \varepsilon}=\frac{\operatorname{Re}}{1+\operatorname{Re} v_{t} / \sigma_{\varepsilon}}, \quad R_{e T}=\frac{1}{1 /\left(\operatorname{Re} P_{r}\right)+v_{t} / P_{r t}}
$$

where Re is Reynolds number with $\operatorname{Re}=\frac{v_{j} d}{v}$ and $v_{t}=C_{\mu} \frac{k^{2}}{\varepsilon}$. In equation (5) and (6):

$$
P_{k}=v_{t}\left[2\left(\frac{\partial u}{\partial x}\right)^{2}+2\left(\frac{\partial v}{\partial y}\right)^{2}+2\left(\frac{\partial w}{\partial z}\right)^{2}+\left(\frac{\partial v}{\partial x}+\frac{\partial u}{\partial y}\right)^{2}+\left(\frac{\partial v}{\partial z}+\frac{\partial w}{\partial y}\right)^{2}+\left(\frac{\partial w}{\partial x}+\frac{\partial u}{\partial z}\right)^{2}\right]
$$

Constants in the model are as following:

$$
\begin{aligned}
& C_{\mu}=0.085, \quad C_{\varepsilon 1}=1.42-\frac{\eta\left(1-\eta / \eta_{0}\right)}{C_{\varepsilon 2}}, \quad \eta=1.68, \quad \sigma_{\mathrm{k}}=0.7179, \quad \sigma_{\varepsilon} \underline{\underline{\eta}}_{0.7179}^{3}, S=\left(P_{k} / v_{t}\right)^{1 / 2}, \beta=0.015, \\
& C_{\varepsilon=1},
\end{aligned}
$$

$\Delta T=(T-1) t_{a}$ is extra temperature and $t_{a}$ is local environmental temperature. $\beta$ is thermal coefficient of expansion of water and a function for it proposed by Batchelor $(1970)^{[8]}$ as following:

$$
\beta(t)=\left(-0.773+0.19 t-0.0027 t^{2}+0.000021 t^{3}\right) \times 10^{-4}
$$

in which $t$ is local temperature with its unit of degree Celsius. 


\section{Boundary Conditions}

A vertical buoyant jet from a square orifice located on a bed in semi-infinite static ambient was simulated and boundary conditions for six boundary surfaces with exit temperature $t_{j}$ as following:

Bottom boundary with $y=0$ :

$u=0, v=1, w=0, \quad T=t_{j} / t_{a}, \quad k_{0}=0.006, \quad \varepsilon_{0}=0.0001$ at the exit of the orifice;

The wall functions was used to relate the values of $u, w 、 k 、 \varepsilon$ at the first grid points outside the viscous sub layers to boundary conditions with $v=0$ and heat insulation was applied with $\frac{\partial T}{\partial y}=0$ out of the exit.

Left and right boundary: $\quad \frac{\partial u}{\partial x}=\frac{\partial v}{\partial x}=\frac{\partial w}{\partial x}=\frac{\partial k}{\partial x}=\frac{\partial \varepsilon}{\partial x}=\frac{\partial T}{\partial x}=0$

Fore and after boundary: $\quad \frac{\partial u}{\partial x}=\frac{\partial v}{\partial x}=\frac{\partial w}{\partial x}=\frac{\partial k}{\partial x}=\frac{\partial \varepsilon}{\partial x}=\frac{\partial T}{\partial x}=0$

Upper boundary: $\quad \frac{\partial u}{\partial y}=\frac{\partial v}{\partial y}=\frac{\partial w}{\partial y}=\frac{\partial k}{\partial y}=\frac{\partial \varepsilon}{\partial y}=\frac{\partial T}{\partial y}=0$

\section{Method of Calculation}

The uniform format deduced from above partial differential equations (2) (7) in the three dimensional mathematical model can be written as following:

$$
\frac{\partial^{2} \Phi}{\partial x^{2}}+\frac{\partial^{2} \Phi}{\partial y^{2}}+\frac{\partial^{2} \Phi}{\partial z^{2}}=2 A \frac{\partial \Phi}{\partial x}+2 B \frac{\partial \Phi}{\partial y}+2 C \frac{\partial \Phi}{\partial z}+G
$$

Hybrid finite analytic method was used to solve the equation (13). The stagger grid was applied to avoid improper sawtooth-like pressure distribution arisen probably from calculating all the variables on a same node. Non-uniform grid distribution was employed for calculation. The size of calculated domain and number of grid would be adjusted lightly according to different conditions. Convergence is declared when the normalized residual is less than $1 \times 10^{-3}$ for equations of $u, v, w$ and $1 \times 10^{-5}$ for others. Programs for calculations were written in FORTRAN language.

\section{Velocity by Numerical Simulations}

Fig. 1 shows axial velocities under different Froude numbers with vertical coordinate $v_{m} / v_{j} \cdot F_{0}$, in which $v_{m}$ and $v_{j}$ denotes axial and exit velocity. Formulas of axial velocity for a round buoyant jet proposed by Chen and Rodi (1976) and Noutsopoulos and Yannopoulos (1987) were also shown in Fig.1. It shows that axial velocities of jets from a square orifice follow the line calculated by the formulas for those of jets from a round orifice after a distance from exit. $K_{w}$ in the formula by Noutsopoulos and Yannopoulos (1987) is as following:

$$
K_{w}=0.12-0.02 \exp \left(-0.05 Y^{2}\right)
$$




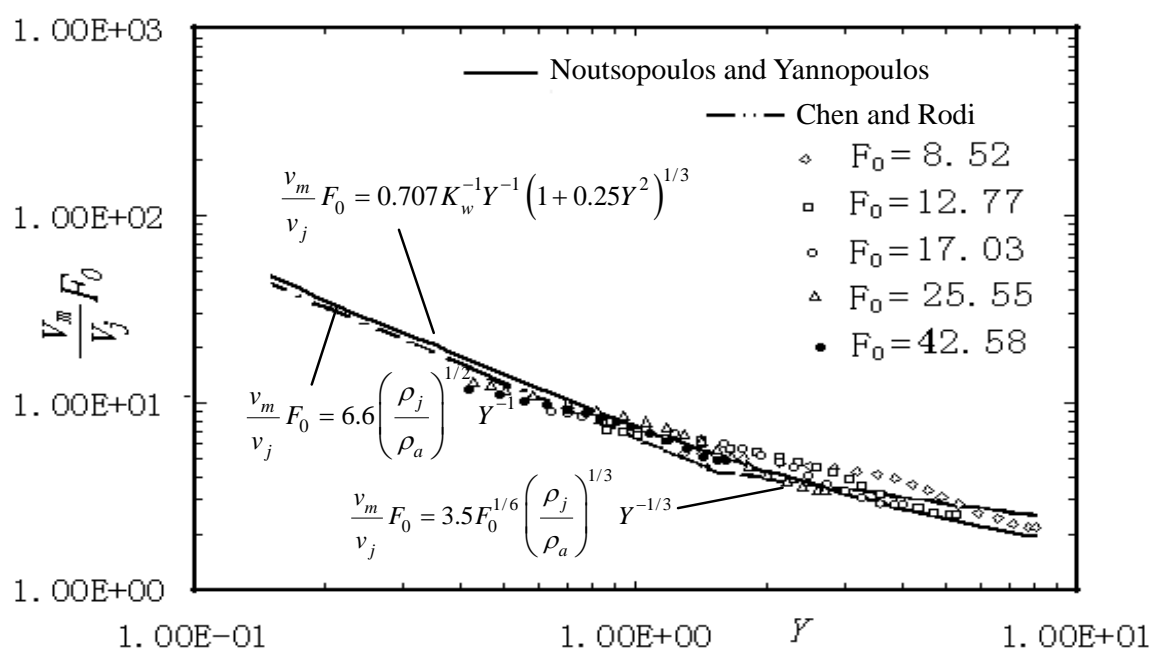

Fig.1 Axial velocity distribution law

Contours of speed on cross sections with different height from exit under Froude number $F_{0}=12.77$ were displayed in Fig. 2 by numerical simulations. It shows that the shape of contours changes rapidly from polygon to concentric circular after a short distance from exit, also completely similar to those of vertical buoyant jet from a round orifice.
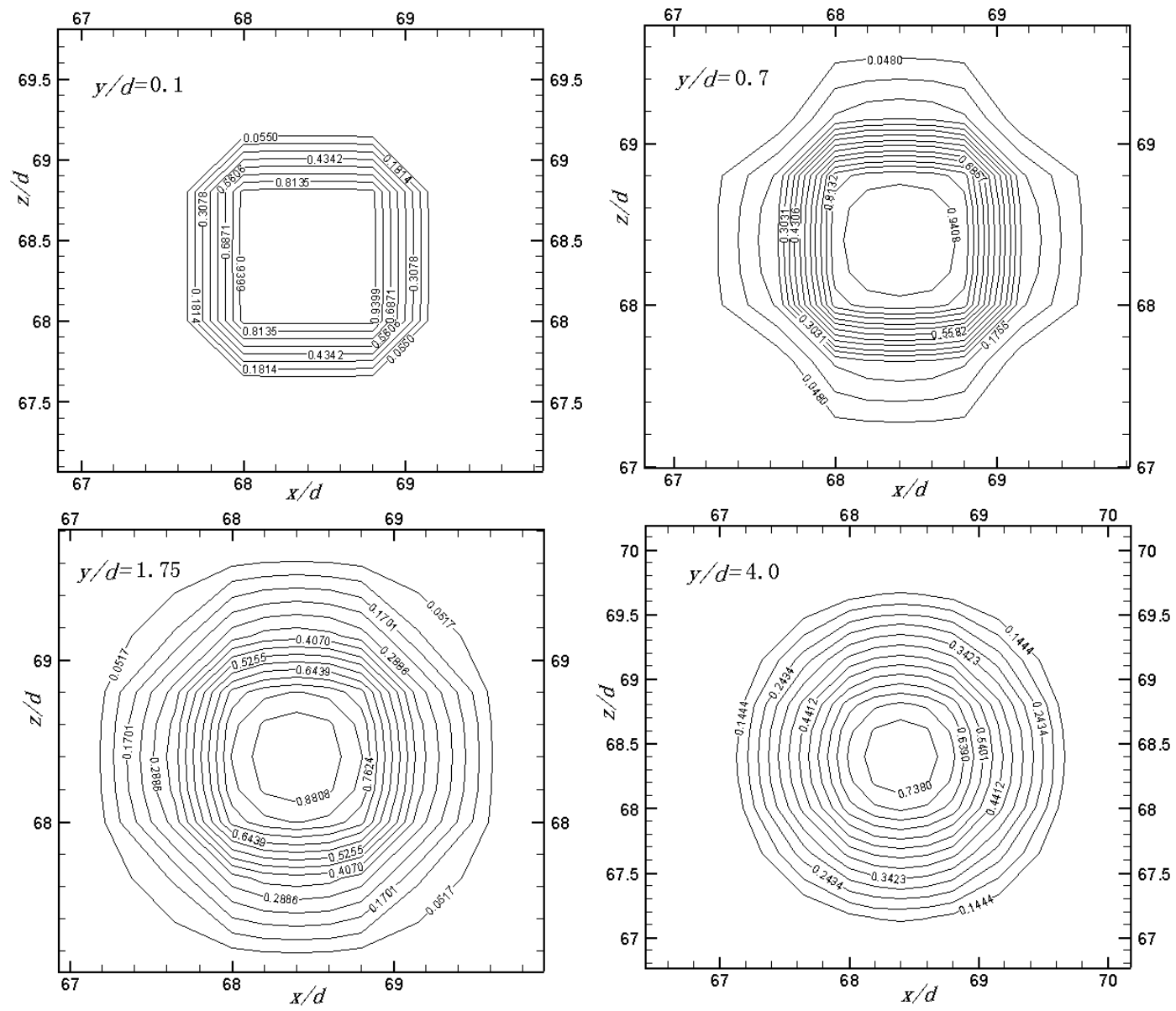

Fig.2 Velocity contours of $v$ on different heights for $F_{0}=12.77$

The characteristic of self-similarity of speed on a cross section was also validated in Fig.3, which shows values of vertical velocity $v$ on a line intersecting with the axis of jet on a cross section with different heights under Froude number $F_{0}=8.52$. The vertical distance from exit required to reach flowing area of self-similarity increases with increasing of Froude number. However, flowing area affected on a cross section was limited to $-0.2 \leq x / y \leq 0.2$ irrespective of Froude number. 


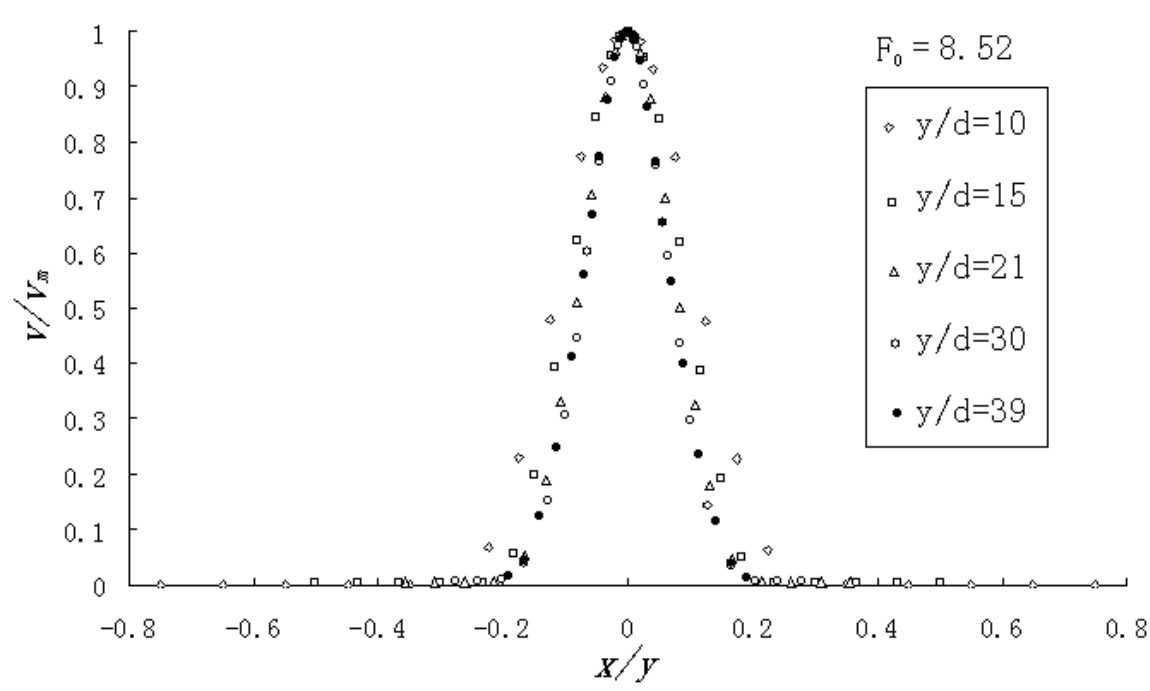

Fig.3 Velocity $v$ distribution on different cross sections for $F_{0}=8.52$

\section{Conclusions}

Thermal water buoyant jet from a square orifice in a semi-infinite static ambient was studied by using the methods of three-dimensional mathematical model. Axial velocities under various density Froude number have been gained. Efficiency of the mathematical model was validated by well coincidence of results from numerical simulations. Axial velocity of vertical buoyant jets from a square orifice were compared with those calculated by the formulas for a round orifice proposed by previous engineers. It shows that axial velocity from a square orifice can be predicted by these formulas existed for a round vertical buoyant jet.

The characteristic of self-similarity of speed on a cross section with various heights after a short distance from exit has also been validated for vertical buoyant jet from a square orifice, which is also completely similar to those from a round one.

\section{References}

[1] List E J. 1982. Mechanics of turbulent buoyant jets and plumes: In Turbulent Buoyant Jets and Plumes, Pergamon, pp: 1-68.

[2] Chen C J, Rodi W. 1980. Vertical turbulent buoyant jets: A review of experimental data, HMT, Series 4, Pergamon Press, Oxford.

Chen C J, Rodi W. 1976. A review of experimental data of vertical turbulent buoyant jets, Report No. 193, Iowa Inst. of Hydr. Res.

[3] Jirka G H, Abraham G, Harleman D R F. 1975. An assessment of techniques for hydrothermal prediction, Report No.203. M.I.T., Ralph M. Parsons Lab.

[4] Fischer H B, List E J, Imberger J, Brooks N H. 1979. Mixing in inland and coastal waters, Academic Press, New York.

[5] Noutsopoulos G C, Yannopoulos P C. 1987. The round vertical turbulent buoyant jet, Journal of Hydraulic Research, 25(4):481 502

[6] Huai Wenxin, Fang Shenguang, Dai Huichao. 2006. Behavior of obstructed squared buoyant vertical jets in static ambient (I)-Verification of mathematical model and numerical method, Applied Mathematics and Mechanics, 27(5):645 652

[7] Huai Wenxin, Fang Shenguang. 2006. Rounded flowing states of obstructed buoyant jet, Applied Mathematics and Mechanics, 27(8):1133 1139 
[8] Batchelor G K. 1970. An introduction to fluid dynamics, Cambridge University Press, Great Britain. 